\title{
Auto-evaluating System of Computer Hardware Experiment Based on Remote Hardware Platform
}

\author{
Li Shanshan and Quan Chengbin
}

\begin{abstract}
For the requirement of the Massive Open Online Courses, computer hardware experiment teaching should support online and massive experiment. This paper demonstrates the computer hardware online experiment system based on the real hardware experiment platform, and it can evaluate the experiment design automatically. This system explores an effective practice method for the massive online computer hardware experiment.
\end{abstract}

Index Terms-MOOC, massive online auto-evaluate, computer hardware experiment, cloud experiment.

\section{INTRODUCTION}

In the computer curriculum system, hardware courses are the important part as the software courses [1]. Hardware courses include Digital Logic, Computer Organization, Computer Architecture, etc. These courses all have an important feature that their theoretical knowledge is based on the real hardware, so these courses need the strong practical experiments for helping students to understand the content of the courses. The experiment teaching takes an important part of these courses, and it can develop students' practical ability, engineering ability and innovative ability [2], [3]. Therefore, the teaching process in various colleges and universities involves the experiment teaching in hardware course as an important composition.

\section{LARGE-SCALE ONLINE EXPERIMENTS}

MOOC(Massive Open Online Courses) is carried out in full swing now, and it has become an important area for education in future. Many colleges and universities have online some computer courses on their MOOC system and achieved the good results, but these courses are mainly theoretical teaching, lack of appropriate experimental teaching, which is mainly due to the limitation of the experimental teaching for the large-scale online experiments. For the experimental teaching, how to carry out large-scale online experiment is the important issue in current teaching in MOOC [4]. The main encountered difficulties are the following two aspects:

One is how to deploy the online experiment. Because online learners are using remote learning, they do not have the conditions to do the experiments in the laboratory, so the experimental teaching must also be completed online. For the

Manuscript received April 6, 2015; revised September 22, 2015

The authors are with the Department of Computer Science and Technology, Tsinghua University, Beijing, 100084 China (e-mail: lishanshan@tsinghua.edu.cn, quancb@tsinghua.edu.cn). software experiments, the situation is better, because the experiments are just need the appropriate software environment, and the learner can connected to the experimental server remotely to complete the experiments. But hardware experiments require some specific equipment, the experimenters are hard to do the experiment in lack of these equipment. Thus the hardware experiments need some technological methods to solve this problem, such as software simulating, or special experimental system remotely using experiment equipment.

The other is the issue of large-scale experiments. Compared with the traditional experiments, the number of the experimenters in the large-scale experiment is much more than ever, which resulted that the experiment deployment and check become a difficult problem. Using the traditional manual mode is certainly not a realistic solution. The only method is the experimenter doing the experiments independently and computer automatically experiments verification. Thus the large-scale experiments can be carried out smoothly. Currently the software courses, such as Computer Programming, have begun using the online evaluation system to assist the experimental teaching [5], [6]. Through years of use, this is proved to be a feasible way in software course experiments, and it ensured the experimental results and significantly improved the efficiency in experiments checking.

For computer hardware experimental teaching, the above two issues are particularly prominent. Above all, how to launch the online experiments. As already mentioned, one solution is the virtual laboratory, using software to simulate the hardware to run experiments, but the main drawback of this solution is that the software emulation can not simulation the real hardware running very well. It is not fully simulating the real experimental process, so it causes the experimental design in this way is perhaps not correct on real hardware devices.

Another solution is using the remote experiment system based on real hardware. There are several sample experiment systems developed by some colleges and universities, such as Tsinghua University, National University of Defense Technology. These experiment systems are based on real hardware experiment devices, in which the experimental data is sampled and transferred through the network, so as to achieve the purpose of remote experiment [7]. Thus the experimenter can operate the experiment devices remotely, just same as local experiment.

And for the issue of the experiment automatic checking or validation, the experimental devices can be set the input stimulus and its output result also can be sampled in the experiment procedure, so the experiment can be verified through comparing the sampled result with the correct result 
data stored in advance. Thus the issue of automatic evaluation is solved.

Based on the above considerations, we developed and implemented a remote computer hardware experiment and auto-evaluation system, on which we can do the computer hardware experiments online. And this system provides the automatic verification for each experiment, so it can be the basis platform for the future large-scaled online experiment.

\section{SYSTEM ARCHITECTURE AND EXPERIMENT FLOW}

The traditional custom hardware experimental equipment has fixed structure and the experiments on it are also fixed and limited. The experiments on it are lack of scalability, flexibility and innovation, so these experimental apparatus are difficult to apply to the large-scaled online experiment. Meanwhile the application of large-scaled programmable logic devices in experiment makes the hardware experiment and evaluation possible in large-scaled online experiment. The experimenter can use the local EDA software to complete the experiment design, then he can configure the experimental programmable device remotely via a network and the experiment can be carried out on it. In the experimental process the experimenter does not care about the detailed architecture of the experimental system, only focus on the experiment. The programmable devices provide rich peripheral pins, through assigning these pins to the concerned signals, the experimental system can get the experiment data in the whole process of the experiment [8], which provides the convenient for subsequent experimental evaluation. Thus using programmable devices in the experiment is the basis for the online computer hardware experiment and evaluation.

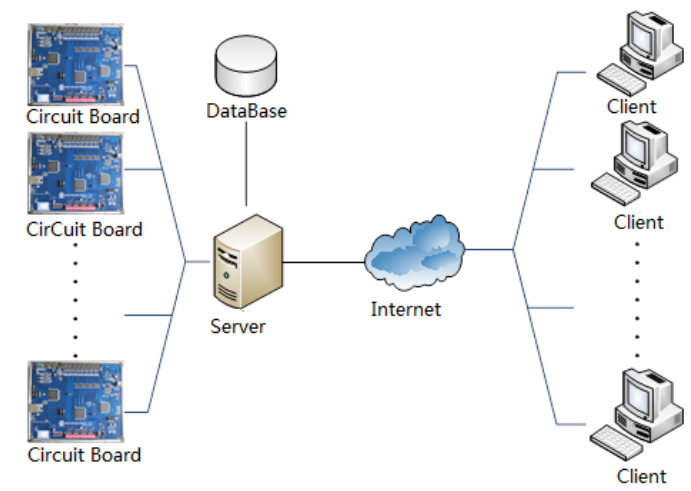

Fig. 1. Structure of the hardware online experiment and evaluation system.

The structure of the hardware online experiment and evaluation system is shown as Fig. 1. The experiment circuit board is the Computer Hardware Unified Platform [9] which is a computer hardware experiment equipment designed by DCS\&T Tsinghua University, and it supports computer main hardware courses' experiments. This experiment platform uses the large-scale programmable device as the main experimental chip, and has the control circuit to set or sample the experiment data. The experiment server manages the experiment circuit board and provides the experiment service for the clients. The database stores the experimental content and the test data and standard test result for the experiments. The client software provides the user interface for the experimenters to accomplish the experiment operation.

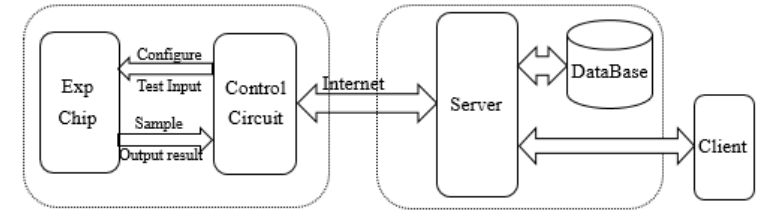

Fig. 2. Data path

In the actual experiment, the experimenter uses the client software to connect to the server, and applies an experiment circuit board. The server will response it and assign a hardware circuit board to this experimenter. The experimenter will finish the experiment design and testing on this board using the user interface in client software. When the experimenter completes his design, the server will automatically evaluate this design using the test data or standard result of the corresponding experiment stored in database, so as to check whether the experiment is successful. For each experiment process, the server will record the intermediate experimental data in the database. The data path of the experiment system is shown as Fig. 2.

The detailed experiment and evaluation process is as followings:

1) The experimenter chooses the experiment in client software, and completes the experiment designed according to the requirement in the experiment content.

2) The experimenter connected to the server and is assigned to a hardware circuit board, then he can submit the experiment design file to the server.

3) The server will send the design file to the corresponding experiment circuit board, and the control circuit on this circuit board will configure the design file to the experimental chip which is a programmable device, thus the experiment design is running on the experimental chip.

4) The experimenter can set the input stimulus through the interface of the client software, and the server will return the output result to client, so the experimenter can check whether the experimental result is same as expected. In this process, the experimenter can debug his design with the help of the client.

5) If the experimenter completes his testing, he can submit the final experiment design to the server, and the server will auto-evaluate this design.

6) The server will import the test cases from the database according to the content of the experiment, and send these test cases to the control circuit. According to the test case, the control circuit gives the experiment chip the input test signals and obtains the output signals as the test results.

7) The control circuit will send results back to the server, the server will compare these results with the correct results of the test case stored in the database to determine the current experimental design is correct for this test case. If this test cases passed, test the next test case until all the test cases passed.

The flow chart of the experiment and evaluation is shown as Fig. 3. In this experiment procedure, the teacher do not need to participate. The experimenter can successfully complete the online experiment design and evaluation. Experiment evaluation process is done automatically, which 
saves the workload of the teacher and improve the efficiency of the experimental examination. And the experimental evaluation uses a large number of test cases, which improves reliability of the evaluation in computer hardware experiment.

\section{System FEATURES AND PROBLEMS}

Online experiment and automatic evaluation makes it possible that the computer hardware experiments can be applied to large-scale online experiments. In the entire system, the circuit boards are the hardware experimental basis. The number of the circuit board determines the number of online experiments at the same time. Because the online experimenters on the experiment are distributed in time, and in the whole experiment procedure the experimenter occupies the circuit board only for a short period, so the server needs to manage the circuit board well, releases the un-used hardware resources (e.g. using Heartbeat Algorithm), thus it need a small amount of experiment devices that can support a large number of experiments well [10]. Here we also refer to the concept of "cloud", integrating the experimental platform at different locations to form an experimental cloud, enhancing the scale and efficiency of hardware devices.

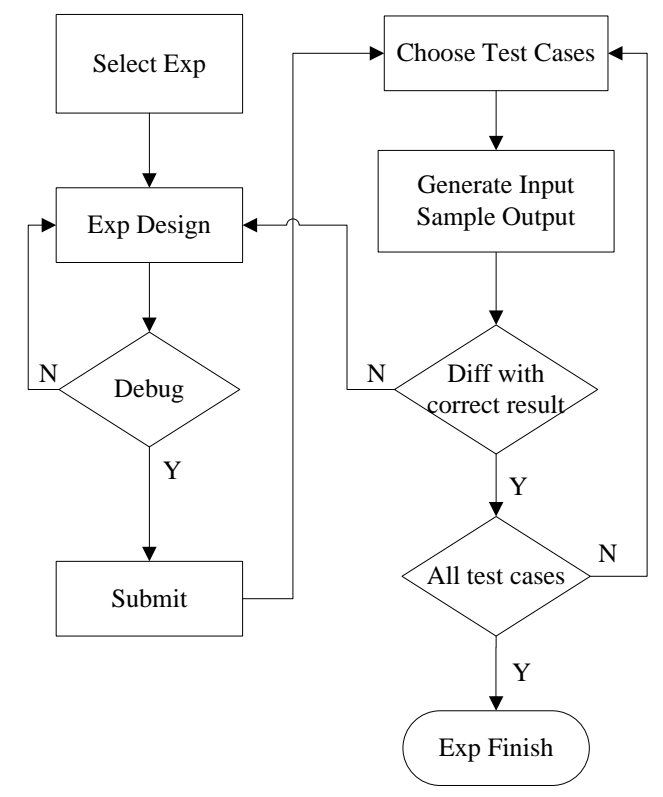

Fig. 3. Experiment and evaluation flow.

In order to ensure the reliability of the experimental evaluation in the experimental system, the system provides sufficient test cases for every experiment, covering the major and key test points on the corresponding experiments, and in additional the test also includes some cases in critical situations or error-prone cases. All these test cases are stored in the database of the server and each test case is consisted of two main parts, test stimulus waveforms of input and the standard correct waveform of the output. For the simple combinational logic circuit experiment, test case contains a truth table of all the cases, so the evaluation of these experiments can be done for full coverage test. While for the sequential logic circuit experiments, the test will use many various test cases, in order to coverage the different timing state testing. For some particular experiments, they have some special cases different from other, such as memory access experiment. For this experiment's one test case, the experiment result is the date stored in the memory section, so that the correct experimental result of this case is a piece of memory data stored in the database. Each of these experiments' cases need the server to do some data processing in the evaluation process.

The software architecture of the experimental is based on CS (Client/Server) mode approach, which can support various experimental content well, but its flexibility is weak. The user need to install the client software locally and when the experiment contents were modified, the user perhaps need to update his software on client. Currently, we are developing BS (Browser/Server) mode solution, as long as the browser can easily fit for the experiment.

On the other hand, due to the large-scale experiments increasing, the hardware resource may insufficient, so the software simulation on the server side can be a good supplement. Let the experimenters solve part of the problems first in the simulator, then let them use real hardware circuit board to do the experiment, thus to reduce the stress of hardware resources and to improve the hardware utilization. In the meanwhile, the new test cases are added to the database continuously as the system providing the experiment service, which will improve the coverage of the test.

In this experiment system, there are many issues that need to be improved, such as how to solve the special needs of the experiment. For example, the peripheral interface experiment such as VGA, serial, etc., how to deploy these experiments, how to display the contents of these interface, how to auto-evaluate these experiments and so on.

This experiment system has been used for trail in undergraduate students of the computer department of Tsinghua University. The students did the experiments of the related courses online and completed the evaluation on this system, and then went to the laboratory for approval of the corresponding experiments. In the attempt of two semesters, the student number and experiment amount are increased. This system achieved good experimental teaching effect, and at the same time, it greatly reduced the laboratory experimental work pressure.

\section{SUMMARY}

The presence of the MOOC greatly promoted the development of the online education, but also brought new demand for the experimental teaching. The experiment system with remote experiment, online, automatic evaluation is the basis of the large-scale online experiments. For computer hardware experiments, the remote experiment system based on real hardware is the future direction of the online experiment. This paper brings an experimental system which tied to solve the problems encountered in the large-scale computer hardware online experiment. The experiment system provides a tentative solution for the computer hardware experiment in MOOC.

\section{REFERENCES}

[1] F. L. Chen and X. M. Qi, "Construction and implementation of innovation driven hierarchical computer hardware courses," University Education, pp. 40-42, Feb. 2013. 
[2] M. Qi, P. Zhang, and Y. Dong, "Research and innovation of hardware experiment teaching," Experiment Science and Technology, pp. 80-81, April 2011.

[3] H. D. Ma and K. J. Bao, "Reform and practice of experimental teaching of computer hardware," Research and Exploration in Laboratory, pp. 360-362, Oct. 2013.

[4] M. Y. Wu and M. Y. Guo, "Discussion on MOOC and future education trend," Computer Education, pp. 5-8, Oct. 2013.

[5] Q. Lin and J. N. Han, "Research in practice teaching of C language based on online judge system," Experiment Science and Technology, pp. 37-39, Dec. 2011.

[6] H. B. Zhang, "Design and implementation of the open cloud platform based open source online judge system," Computer Science, pp. 339-343, Nov. 2012.

[7] H. Y. Lu, Y. H. Tang, and H. Yu. "Computer hardware 'experiment cloud'," Computer Education, pp. 36-38, May 2014.

[8] T. J. Xiao, S. B. Shi, and J. Shen, "Design and implementation of computer hardware experiment system based on JTAG," Data Communications, pp. 24-28, Feb 2013.

[9] C. B. Quan, X. P. Guan, and S. S. Li. "Design of union platform of computer hardware experiment," Computer Education, pp. 3-5, Sep. 2008.

[10] L. J. Yang and H. Y. Lu. "Implementation of computer hardware remote laboratory based on cloud computing platform," Huazhong Univ. of Sci\&Tech. (Natural Science Edition), pp. 72-76, Dec. 2013.

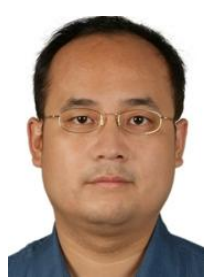

Li Shanshan was born at Shandong China on May 26, 1979. He was graduated from the Department of Computer Science and Technology, Tsinghua University, Beijing, China. He got the master degree of computer architecture in 2005.

$\mathrm{He}$ is working at the Department of Computer Science and Technology, Tsinghua University since graduated and his main work is computer experiment teaching and experiment equipment development.

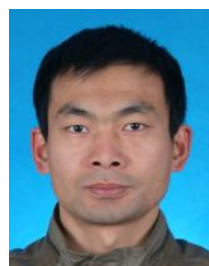

Quan Chengnin was born at Liaoning China on January 28, 1972. He was graduated from the Department of Computer Science and Technology, Tsinghua University, Beijing, China. He got the Ph.D of computer network in 1997.

$\mathrm{He}$ is working at the Department of Computer Science and Technology, Tsinghua University since graduated, and his main work is computer network and experiment equipment development. 